\title{
Investigating the Success of Cover Flap in Patients with Bedsore
}

\author{
Shokoufe Mortazavi ${ }^{1}$, Gholamreza Amirian ${ }^{2}, \&$ Mohammadbagher Heidari $^{2}$ \\ ${ }^{1}$ General Surgeon in Kermanshah University of Medical Sciences, Iran \\ ${ }^{2}$ Plastic and Reconstructive Surgeon in Kermanshah University of Medical Sciences, Iran \\ Correspondence: Shokoufe Mortazavi, General Surgeon in Kermanshah University of Medical Sciences, Iran. E- \\ mail: Dr.mortazavi7@gmail.com
}

Received: May 20, 2019

Accepted: September 26, 2019

Online Published: October 23, 2019

doi:10.5539/jmbr.v9n1p119

URL: https://doi.org/10.5539/jmbr.v9n1p119

\begin{abstract}
Objective: The high prevalence of bedsore creates many problems for the maintenance system and the patients, and in addition to spending high costs for treating ulcers, a lot of time is also dedicated to caring for them. The purpose of performing this study is to investigate the therapeutic results of recovering bedsore injuries by cover flaps.

Work Method: This study is of prospective type in which 85 patients with bedsore who had referred to Taleghani Hospital in Kermanshah for treatment by muscle cover flaps during the years of 2016 to 2017, were followed up at time periods of 1 week, and 3 months after the discharge and in case of failure, they were recovered. Finally, the obtained data were analyzed by using statistical tests and SPSS version 22 software.

Results: The obtained results showed that the success percentage of recovering bedsores was significantly increased after one week, and 3 months by cover flaps $(\mathrm{P}<0.05)$. Also, the success percentage of recovering bedsores by cover flap after one week, and 3 months in terms of age, gender, and BMI of patients significantly shows an increase $(\mathrm{P}<0.05)$.

Conclusion: In general, it can be concluded from this study that using cover flaps leads to the success of recovering bedsores after 3 months of treatment, and the age, gender, and BMI variables of patients cannot be effective in this improvement process.
\end{abstract}

Keywords: Bedsore, Cover Flap, Recovery

\section{Introduction}

Nowadays, health care is very important among all people (Rashid, Hamdi, \& Mustaf, 2018). Bedsore is a global health problem with complex and multifactor causes that are regarded as a disabling complication with high medical costs (Deng, Yu, \& Hu, 2017). Among the main causes of bedsore emergence, features such as sedation, altered consciousness, long-term rest, treatment with mechanical ventilation, special medications, and unstable hemodynamic status can be referred (Cuddigan, 2012). The results of studies have shown that patients suffering from bedsore were generally older and passed a long period of medical treatment (Han, Ko, \& Rhie, 2017). Bedsore, as a secondary problem in patients, increases the burden of illness and causes the patient to face a lot of problems. Despite many efforts that have been made to treat bedsore, bedsore is still a very important and discomforting problem for patients with bedsore, causing harassment and annoyance of patients and lowering the quality of life of these people (van Leen, Schols, Hovius, \& Halfens, 2017; Lechner, Lahmann, Neumann, Blume-Peytavi, \& Kottner, 2017). Additionally, patients suffering from bedsore should pay significant costs for healthcares and treating this illness. The results of various studies have shown that preventive measures reduce the significant ratio of catching bedsores in patients (Dealey, Posnett, \& Walker, 2012; National Pressure Ulcer Advisory Panel/European Pressure Ulcer Advisory Panel.).

Patients, families, treatment and healthcare services centers, and community are significantly affected by the physical, financial and social consequences of pressure ulcers, and patients with pressure ulcers inevitably experience pain, disfigurement, disability and dependence on others (Bansal, Scott, Stewart, \& Cockerell, 2005). The pressure ulcer on average composes $19 \%$ of patients admitted to all hospitals. The prevalence of bedsore in the orthopedic wards in the United States has been reported 10.6\% and in the Tehran orthopedic wards has been reported 25\% (Paryad, Jahanshahi, Jafroodi, \& Kazem Nezhad, 2005). Annually 1,600,000 people in American treatment centers suffer from pressure ulcer; the emergence ratio of this complication is $10 \%$ in patients admitted 
to general hospitals. In Iran, it is estimated that $5 \%$ of patients admitted to the hospitals and $38 \%$ of the clients of recovery places are suffering from pressure ulcer (10). The prevalence of bedsore in the Intensive Care Unit (ICU) has varied from $14 \%$ to $41 \%$, and the emergence of this phenomenon has varied from 1 to $56 \%$, which is two to three times higher than the estimated emergence and prevalence figure in other wards of the hospital (Paul, Keller, $\&$ Wille, 2002). Inappropriate diagnosis, neglecting to perform the pressure ulcer care plan and deficiency in skin care followed by the damage and the lack of anti-pressure equipments are the most important causes of this phenomenon. In addition, despite new methods and tools for the pressure ulcers care, knowledge and performance of physicians in this field are not updated (10).

The results of studies have shown that the use of cover flap can be very useful in the management of bedsore. The effectiveness of this intervention has been reported both in children and in adults (Firriolo, Ganske, Pike, Caillouette, Faulkner, \& Upton, 2018). Also, the results of studies by Gargano et al. (2017) showed that the Cone of Pressure (COP) flap significantly reduces recurrences, eliminates shearing forces, suture ripping, and tension on superficial soft-tissue layers. Large defects in the scalp area are usually treated by plastic surgeons. The restoration of such lesions depends on their location, size and depth. Regarding major defects of head and neck area, although all recovering methods are applicable, the use of skin graft such as applying flap is considered as the most appropriate of these methods, both in terms of beauty and in terms of performance. However, older patients with poor general health, or advanced malignancies or numerous illnesses are not considered as appropriate candidates for long-term treatments and multi-stage surgeries and require more reliable and more trustable treatments that are performed faster (Rubayi \& Chandrasekhar, 2011). Adhesions after surgery are a common cause of surgical morbidity. Adhesions change normal anatomic relationships, which can lead to pain and necessitate additional corrective surgeries (Ebrahimian, Tahmasbi, Bananzadeh, Nasr Esfahani, Nadri, \& Abbaszadeh, 2018). Since few studies have been performed about the use of cover flap to recover bedsore, also, regarding the failure to recover bedsore after surgery, this study was conducted aiming to investigate the therapeutic results of recovering bedsore injuries by cover flap in Kermanshah during the years of 2016-2017and its role in maintaining the organ and also the emergence of complications related to these treatments.

\section{Materials and Methods}

This study was of prospective type and the population under study included patients with bedsore, who had referred to Taleghani Hospital for treatment by muscle cover flaps during the years of 2016-2017. Sampling method in this study was by available method. The sample size ratio was calculated by using the studies of Yang et al. (2011) and the therapeutic success ratio variable was calculated and the value of 85 people was considered. Therefore, the total number of samples was regarded as equal to 85 people. In this study, patients were also investigated in respect of the presence of the underlying diseases that were effective in performing the study and the results of recovering treatment and in case of existence of diseases such as human immune system defect and diabetes, these patients were excluded from the study. As a result, all patients' information that had been treated with a variety of cover flaps were examined by the colleague assistant of the plan on the basis of the file information and at regular time intervals, and the results were recorded in the corresponding checklist. In these patients, the cover flaps were investigated with respect to the efficiency and also the function of related organ, as well as the complications ratio and failure of the above treatment, and after the final investigation, the results were presented. In the next stage, the patient's condition was followed up to 1 week, and 3 months after discharge, they have recovered again in case of failure (lack of approximation of the recovering location to the normal state).

\section{Data Analysis}

After recording the data in the checklist, encoding the data was performed and it was transferred to SPSS version 22 software. Descriptive statistics (mean, and one-dimensional and two-dimensional tables and diagrams, standard deviations and variance) were exploited to summarize the data. For the analysis of quantitative data based on KS test, the normality of data was investigated. Then, an independent t-test or Mann-Whitney $U$ test was used. For qualitative data, Chi-square test or Fisher's exact test was also used. The significance level was considered equal to 0.05 .

\section{Findings}

In this study, 85 patients with bedsore who had referred to Taleghani Hospital in Kermanshah for treatment during the years of 2016-2017 and had the criteria to enter the study were investigated. The age of patients ranged from 28 to 43 years old with a mean and standard deviation of $33.61 \pm 3.96$ years. In terms of gender, 54 people $(63.5 \%)$ were male and 31 people (36.5\%) were female. Also, 49 people $(57.5 \%)$ had BMI $<=25$ and 36 people (42.5\%) had BMI> 25. Considering the frequency values of bedsore type, 52 people $(61.2 \%)$ had ischial bedsore, 20 people $(23.5 \%)$ had sacral bedsore, 10 people $(11.8 \%)$ had trochanteric bedsore, and 3 people $(3.5 \%)$ had heel bedsore. 
Considering the frequency values of flap type, 52 people (61.2\%) had upper gluteus pedicle flap, 20 people (23.5\%) had V-Y flap, 10 people (11.8\%) had Lacteral Thigh flap, and 3 people (3.5\%) had Advanced flap. Investigating the frequency values of complications type of patients also showed that 77 people $(90.5 \%)$ had no complications, 4 people (4.7\%) had partial necrosis complications, 2 people (2.4\%) had complete necrosis, and 2 people (2.4\%) had seroma.

In this study, Wilcoxon test was used to compare the success percentage of recovering bedsores by cover flaps after 3 months. The frequency values and comparison of the success percentage of recovering bedsores by cover flaps after 3 months have been summarized in Table 1 .

Table 1. Frequency Value and Comparison of the Success Percentage of Recovering Bedsores by Cover Flaps after 3 Months

\begin{tabular}{|c|c|c|c|c|}
\hline \multirow{2}{*}{ Measurement Period } & \multicolumn{2}{|c|}{ Treatment Result } & \multirow{2}{*}{ Test Statistics } & \multirow{2}{*}{ P-Value } \\
\hline & Unsuccessful & Successful & & \\
\hline 1 Week & $42(49.4 \%)$ & $43(50.6 \%)$ & \multirow{2}{*}{4.11} & \multirow{2}{*}{$<0.001$} \\
\hline 3 Months & $8(9.5 \%)$ & $77(90.5 \%)$ & & \\
\hline
\end{tabular}

Based on the results of Table (1), no significant difference was observed between the success percentage of recovering bedsores by cover flaps after 1 week, and 3 months $(\mathrm{P}<0.05)$. Thus, the success percentage of recovering bedsores by cover flaps after 3 months showed an increase.

The Wilcoxon test was used to compare the success percentage of recovering bedsores by cover flaps after 3 months in respect of age. The frequency values and comparison of the success percentage of recovering bedsores by cover flaps after 3 months in terms of age have been summarized in Table (2).

Table 2. Frequency Values and Comparison of the Success Percentage of Recovering Bedsores by Cover Flaps in Patients with Less than 33 Years Old, after 3 Months

\begin{tabular}{llcr}
\hline \multirow{2}{*}{ Measurement Period } & Treatment Result & Test Statistics \\
\hline 1 Week & Unsuccessful & Successful & $16(42.1 \%)$ \\
3 Months & $22(57.9 \%)$ & $27(71.1 \%)$ & 2.52 \\
\hline
\end{tabular}

Based on the results of Table 2, a significant difference is observed between the success percentage of recovering bedsores by cover flaps in patients with less than 33 years old after 1 week, and 3 months $(\mathrm{P}<0.05)$. Thus, the success percentage of recovering bedsores by cover flaps in patients with less than 33 years old after 3 months shows an increase. Also, the frequency values and comparison of the success percentage of recovering bedsores by cover flaps in patients with 33 or higher years old after 3 months showed that a significant difference is observed between the success percentage of recovering bedsores by cover flaps in patients with 33 or higher years old after 1 week, and 3 months $(\mathrm{P}<0.05)$. Therefore, the success percentage of recovering bedsores by cover flaps in patients with 33 or higher years old after 3 months increases (Table 3 ).

Table 3. Frequency Values and Comparison of the Success Percentage of Recovering Bedsores by Cover Flaps in Patients with 33 or Higher Years Old after 3 Months

\begin{tabular}{llrlr}
\hline \multirow{2}{*}{ Measurement Period } & \multicolumn{2}{c}{ Treatment Result } & \multirow{2}{*}{ Test Statistics } & P-Value \\
\hline 1 Week & Unsuccessful & Successful & \multirow{2}{*}{0.001} \\
3 Months & $20(42.6 \%)$ & $27(57.4 \%)$ & 3.3 & \\
\hline
\end{tabular}

The Wilcoxon test was used to compare the success percentage of recovering bedsores by cover flaps after 3 months in terms of gender. The frequency values and comparison of the success percentage of recovering bedsores by cover flaps after 3 months in terms of gender have been summarized in Table (4). 
Table 4. Frequency Values and Comparison of the Success Percentage of Recovering Bedsores by Cover Flaps in Female Patients after 3 Months

\begin{tabular}{llcr}
\hline Measurement Period & Treatment Result & Test Statistics \\
& Unsuccessful & Successful & $17(54.8 \%)$ \\
1 Week & $14(45.2 \%)$ & $28(90.3 \%)$ & 3.05 \\
3 Months & $3(9.7 \%)$ & 0.002 \\
\hline
\end{tabular}

Based on the results of Table 4, there is a significant difference between the success percentage of recovering bedsores by cover flaps in female patients after 1 week, and 3 months $(\mathrm{P}<0.05)$, and the success percentage of recovering bedsores by cover flaps in female patients after 3 months increases. Also, based on the results of Table (5), there is a significant difference between the success percentage of recovering bedsores by cover flaps in male patients after one week, and 3 months $(\mathrm{P}<0.05)$, and the success percentage of recovering bedsores by cover flaps in male patients after 3 months increases (Table 5).

Table 5. Frequency Values and Comparison of the Success Percentage of Recovering Bedsores by Cover Flaps in Male Patients after 3 Months

\begin{tabular}{llrlr}
\hline \multirow{2}{*}{ Measurement Period } & \multicolumn{2}{c}{ Treatment Result } & \multirow{2}{*}{ Test Statistics } & P-Value \\
\hline 1 Week & Unsuccessful & Successful & \multirow{2}{*}{0.004} \\
3 Months & $26(48.1 \%)$ & $28(51.9 \%)$ & 2.85 & \\
\hline
\end{tabular}

Also, the Wilcoxon test was used to compare the success percentage of recovering bedsores by cover flaps after 3 months in respect to BMI. The frequency values and comparison of the success percentage of recovering bedsores by cover flaps after 3 months in terms of BMI have been summarized in Table 6.

Table 6. Frequency Values and Comparison of the Success Percentage of Recovering Bedsores by Cover Flaps in Patients with BMI less than 25, after 3 Months

\begin{tabular}{llrr}
\hline \multirow{2}{*}{ Measurement Period } & Treatment Result & Test Statistics \\
\hline 1 Week & Unsuccessful & Successful & P-Value \\
3 Months & $18(46.2 \%)$ & $21(53.8 \%)$ & 3.87 \\
\hline
\end{tabular}

Based on the results of Table 6, a significant difference is observed between the success percentage of recovering bedsores by cover flaps in patients with BMI less than 25 after 1 week, and 3 months $(\mathrm{P}<0.05)$, and the success percentage of recovering bedsores by cover flaps in patients with BMI less than 25, after 3 months shows increase. Also, based on the results of Table 7, it shows a significant difference between the success percentage of recovering bedsores by cover flaps in patients with BMI higher than or equal to 25, after one week, and 3 months $(\mathrm{P}<0.05)$, and the success percentage of recovering bedsores by cover flaps in patients with BMI higher than and equal to 25, after 3 months increases (Table 7).

Table 7. Frequency Values and Comparison of the Success Percentage of Recovering Bedsores by Cover Flaps in Patients with BMI Higher than or Equal to 25, after 3 Months

\begin{tabular}{llcc}
\hline \multirow{2}{*}{ Measurement Period } & Treatment Result & Test Statistics \\
& Unsuccessful & Successful & \multirow{2}{*}{ P-Value } \\
\hline 1 Week & $24(52.2 \%)$ & $22(47.8 \%)$ & 2.13 \\
3 Months & $14(30.4 \%)$ & $32(69.6 \%)$ & 0.033 \\
\hline
\end{tabular}

\section{Discussion and Conclusion}

Bedsore is a common but preventable illness that is often observed in high-risk populations and the emergence ratio of this illness is also increasing year by year (Gargano, Edstrom, Szymanski, Schmidt, Bevivino, Zienowicz, Stark, Taylor, Podda, \& Liu, 2017). The treatment of ulcers in these patients varies depending on the depth of the 
ulcer, the degree of destruction, and the associated infections and various created complications (Cushing, 2013). Deepithelialized flaps were used in the past to eliminate dead spaces and reduce shearing forces (Gargano, Edstrom, Szymanski, Schmidt, Bevivino, Zienowicz, Stark, Taylor, Podda, \& Liu, 2017; Erba et al., 2011). It has also been reported about the COP flap that this intervention eliminates dead spaces and tightens soft tissues. In addition, the COP flap reduces tight forces between the upper flap and the lower bone (Gargano, Edstrom, Szymanski, Schmidt, Bevivino, Zienowicz, Stark, Taylor, Podda, \& Liu, 2017). Regarding that no extensive study has been performed about the use of cover flap for recovering the bedsore, and considering the failure to recover the bedsore after surgery operation, this study was conducted aiming to investigate the therapeutic results of recovering bedsore injuries by cover flaps and its role to preserve the organs, as well as the emergence of complications associated with this treatment. Based on the most important results obtained from this study, the success percentage of recovering bedsores by cover flaps after 3 months showed an increase. Also, this success process of recovering bedsores by cover flaps after 3 months in terms of age, gender, and BMI of patients showed an increase significantly. Therefore, it can be said that these variables did not have a negative impact on this success process. Bamba et al. (2017) in their studies obtained results consistent with the present study. They found that the ischial, sacral and trochanteric ulcers were observed in $62.3 \%, 41.7 \%$ and $18.4 \%$ of patients respectively, so they used gluteus flap (62\%) and afterwards V-Y flap (29\%) for treatment. Of course, the successful results after recovering bedsore have still remained as a major challenge, and when providing cover flaps, risk factors should be considered and risk reduction strategies in patients should be performed before recovering the bedsore. In a study by Mostafa et al. (2012), in the results consistent with the present study, ulcer opening happened in 2 patients, that one of them was recovered automatically. However, for the rest, a review was carried out, that it was recurred in 2 people after 5 and 6 months. Eventually, all 2 people recovered under similar treatment methods after 6 months of follow-up and without any recurrence. Therefore, myocutaneous flaps can be considered as the first option for treating ischial pressure ulcers. In a study by Lin et al. (2012), in the results consistent with the present study, they concluded that 4 people had typhoid necrosis and in 3 patients, the ulcer had been opened. One patient died 4 days after the operation and recurrence was observed in 2 patients. The ratio of complications for sacral ulcers was equal to $20.8 \%$ and for ischial ulcers was $54.5 \%$. After recovering and repairing the ulcers exposed to risk, all ulcers were treated thoroughly. In a study by Yang et al. (2011), in the results consistent with the present study, they found that the success ratio of the flaps used to recover bedsores was excellent and it was without any complications in $75 \%$, and $8 \%$ suffered partial necrosis. In a study by Ahluwalia et al. (2009), in the results consistent with the present study, they found that the complications of ischial flap were observed in $16 \%$ of cases with a recurrence ratio of $7 \%$. Major complications of patients were removed and in $15 \%$ of patients the need to recover was observed. Therefore, it can be concluded that both the type of flap and the place of recovering significantly affect the success ratio of covering bedsore by the flap.

Unfortunately, none of antiulcer drugs is without side effects or gives a $100 \%$ curative rate or a complete cure (Althaiban, 2018). Pressure ulcers have created challenges for plastic surgeons. Patients with pressure ulcers usually have lower limb paralysis and are incapacitated, and these conditions delay the ulcer improvement after recovering and its recurrence. Surgical treatment of pressure ulcers of the sacrum area aiming to debridement necrotized tissue is to shear extra bone prominences, transferring sufficient soft and live tissue to cover the defect area. Various surgical methods have been used to cover the defect after surgical shearing of pressure ulcers. The gluteus flap has numerous benefits, including the fact that these flaps have more rotation, and the donor's area defect is usually less than eight centimeters, that this size initially and without any complication can be closed. If the defect area is larger than eight centimeters, double-sided gluteus flap may be needed. Among other benefits of this type of flaps is that it is not necessary to perform skin grafting for secondary defect healing, and also improvement after operation is faster in patients. Considering these benefits, it can be said that the gluteus muscle flap as an island flap can be used for healing the secondary ischaemum ulcer and for healing the ulcers of the sacrum area (Moatamed, Alizadeh Otaghvar, \& Tarahomi, 2017).

One of the strength points of this study was being a semi-experimental type and all patients were operated by an experienced surgeon and by the same method. Although this study had limitations, among which the low number of similar studies that causes the limitation of the possibility of comparison with other studies can be mentioned, so it is suggested that more studies are designed in this field in the future. Also, the control group was not considered for this study, so it is suggested to consider this issue in the future studies.

Finally, according to the above points, based on the visual observation, it can be said that the use of cover flaps causes the success of recovering bedsores after 3 months of treatment. Also, the age, gender, and BMI variables of the patient cannot affect this recovery process. 


\section{Conflict of interests}

The authors declare that there is no conflict of interests regarding the publication of this paper.

\section{References}

Beheshti Zavare, Z., Arab, M., Rashidian, A., \& Golestan, B. (2010). Factors affecting pressure ulcer in the ICU units of Tehran University of Medical Sciences teaching hospitals. Journal of School of Public Health and Institute of Public Health Research, 8(3), 81-92.

Ahluwalia, R., Martin, D., \& Mahoney, J. L. (2009). The operative treatment of pressure wounds: A 10 year experience in flap selection. International Wound Journal, 6(5), 355-8.

Althaiban, M. A. (2018). Antiulcer Potential of Olive Leaves Extract in Gastric Ulcer Induced by Indomethacin in Male Rats: Antioxidant and Anti-Inflammatory Effects. Pharmacophore, 9(6), 57-64.

Bamba, R., Madden, J. J., Hoffman, A. N., Kim, J. S., Thayer, W. P., Nanney, L. B., \& Spear, M. E. (2017). Flap reconstruction for pressure ulcers: An outcomes analysis. Plastic and Reconstructive Surgery Global Open, $5(1)$.

Bansal, C., Scott, R., Stewart, D., \& Cockerell, C. (2005). Decubitus ulcers A review of the literature. International Journal of Dermatology, 44, 805-10.

Cuddigan, J. E. (2012). Critical care. In B. Pieper (Ed.), National Pressure Ulcer Advisory Panel. Pressure Ulcers: Prevalence, Incidence, and Implications for the Future. Washington, DC: National Pressure Ulcer Advisory Panel.

Cushing, P. L. (2013). Evidence based medicine: pressure ulcers. Plast Reconstr Surg., 132, 1720-1732.

Dealey, C., Posnett, J., \& Walker, A. (2012). The cost of pressure ulcers in the United Kingdom. J Wound Care, 21(6), 261-266.

Deng, X., Yu, T., \& Hu, A. (2017). Predicting the Risk for Hospital-Acquired Pressure Ulcers in Critical Care Patients. Crit Care Nurse, 37(4), e1-e11.

Ebrahimian, S., Tahmasbi, S., Bananzadeh, A. M., Nasr Esfahani, F., Nadri, S., \& Abbaszadeh, A. (2018). Study of intra-abdominal adhesion after hernia repair with propylene mesh in comparison with amniotic membrane attached with propylene mesh in an animal model. Entomol Appl Sci Lett, 5(1), 43-46

Erba et al. (2011). Tip anchor flap in decubital surgery. Aest Plast Surg., 35, 1133-1136.

Firriolo, J. M., Ganske, I. M., Pike, C. M., Caillouette, C., Faulkner, H. R., \& Upton, J. (2018). 3rd, Labow BI. Long-term Outcomes After Flap Reconstruction in Pediatric Pressure Ulcers. Ann Plast Surg., 80(2), 159163.

Gargano, F., Edstrom, L., Szymanski, K., Schmidt, S., Bevivino, J., Zienowicz, R., Stark, J., Taylor, H. O., Podda, S., \& Liu, P. (2017). Improving Pressure Ulcer Reconstruction: Our Protocol and the COP (Cone of Pressure) Flap. Plast Reconstr Surg Glob Open, 5(3), e1234.

Han, H. H., Ko, J. G., \& Rhie, J. W. (2017). Factors for postoperative complications following pressure ulcer operation: Stepwise multiple logistic regression analysis. Int Wound J., 14(6), 1036-1040.

Lechner, A., Lahmann, N., Neumann, K., Blume-Peytavi, U., \& Kottner, J. (2017). Dry skin and pressure ulcer risk: A multi-center cross-sectional prevalence study in German hospitals and nursing homes. Int J Nurs Stud., 73, 63-69.

Lin, P. Y., Kuo, Y. R., \& Tsai, Y. T. (2012). A reusable perforator-preserving gluteal artery-based rotation fasciocutaneous flap for pressure sore reconstruction. Microsurgery, 32(3), 189-95.

Moatamed, S., Alizadeh Otaghvar, H. R., \& Tarahomi, M. R. (2017). Gluteus Maximusmyocutaneous Island Flap for Repairing of Sacral Decubitus Ulcers. Journal of Iranian Surgery, 24(4), 20-6.

Mostafa, W. A. (2012). Inferior gluteus maximus myocutaneous flap for reconstruction of ischial bed sores. Egypt J Plast Reconstr Surg., 36, 201-8.

National Pressure Ulcer Advisory Panel/European Pressure Ulcer Advisory Panel. Pressure Ulcer Prevention Quick Reference Guide. Retrieved from http://www.npuap.org/wpcontent/uploads/2012/02/Final _Quick_Prevention_for_web_2010.pdf

Paryad, E., Jahanshahi, M., Jafroodi, S., \& Kazem Nezhad, E. (2005). Survey of the Quality of Care in Provided in Prevention of Pressure Sores in Hospitalized Patients in Orthopedic Wards, 14(53), 36-42. 
Paul, B. J., Keller, A., \& Wille, J. (2002). Bert van ramshort, Chrisian van der werken, Pressure ulcer in intensive care patient: A review of risk \& prevention, intensive care med., 28, 1379-1388.

Rashid, S. A., Hamdi, M. M., \& Mustaf, A. Sh. (2018). Monitoring the Cardiovascular Parameters (HR, RR, PBP) Under Pressure Situation. International Journal of Pharmaceutical Research \& Allied Sciences, 7(2), 191199.

Rubayi, S., \& Chandrasekhar, B. S. (2011). Trunk, abdomen, and pressure sore reconstruction. Plastic and Reconstructive Surgery, 128(3), 201e-15e.

van Leen, M. W., Schols, J. M., Hovius, S. E., \& Halfens, R. J. (2017). A Secondary Analysis of Longitudinal Prevalence Data to Determine the Use of Pressure Ulcer Preventive Measures in Dutch Nursing Homes, 2005 2014. Ostomy Wound Manage, 63(9), 10-20.

Yang, C. H., Kuo, Y. R., Jeng, S. F., \& Lin, P. Y. (2011). An ideal method for pressure sore reconstruction: A freestyle perforator-based flap. Annals OF Plastic Surgery, 66(2), 179-84.

\section{Copyrights}

Copyright for this article is retained by the author(s), with first publication rights granted to the journal.

This is an open-access article distributed under the terms and conditions of the Creative Commons Attribution license (http://creativecommons.org/licenses/by/4.0/). 